\title{
Magnetic Cannon: the physics of the Gauss rifle
}

Arsène Chemin, ${ }^{1}$ Pauline Besserve, ${ }^{1}$ Aude Caussarieu, ${ }^{1}$ Nicolas Taberlet, ${ }^{1}$ and Nicolas Plihon ${ }^{1,}$ a)

Univ Lyon, Ens de Lyon, Univ Claude Bernard, CNRS, Laboratoire de Physique, Département de Physique, F-69342 Lyon, France

(Dated: 13 December 2019)

The magnetic cannon is a simple device that converts magnetic energy into kinetic energy: when a steel ball with low initial velocity impacts a chain made of a magnet followed by a few other steel balls, the last ball of the chain is ejected at a much larger velocity. The analysis of this spectacular device involves understanding of advanced magnetostatics, energy conversion and collision of solids. In this article, the phenomena at each step of the process are modeled to predict the final kinetic energy of the ejected ball as a function of a few parameters which can all be experimentally measured.

\section{INTRODUCTION}

\section{A. What is a magnetic cannon?}

The magnetic cannon, sometimes referred to as the Gauss rifle is a simple device that accelerates a steel ball through conversion of magnetic energy into kinetic en$\mathrm{ergy}^{1-4}$. The energy conversion at work is reminiscent of other electromagnetism-based accelerating device, such as rail-guns ${ }^{5}$. Figure 1 shows a time sequence (from top to bottom) of a typical setup where a line of four balls (the first one being a permanent magnet) is resting on a rail. When an additional ball approaches from the left with a low initial velocity, it experiences an attractive magnetic force from the magnet, collides with the magnet, and the final ball on the right is ejected at high velocity. Note that, to highlight the various sequences in Figure 1, frame-times are not equi-spaced. The video from which these frames have been extracted is provided as a supplementary material. To understand the physics of the Gauss rifle, the process may be divided into three phases: (i) acceleration of the ferromagnetic steel ball in the magnetic field created by the magnet (frames I to III in Figure 1), (ii) momentum propagation into the chain of steel balls which is similar to the propagation in the Newton's cradle (frame IV), (iii) ejection of the final ball escaping the residual magnetic attraction (frames $\mathrm{V}$ and VI). While Figure 1 highlights a specific example, we will focus on the more general case described in Figure 2, where the initial chain is formed of $n$ steel balls in front of the magnet and $m$ balls behind (each ball of mass $M$ having a radius $R$ ). The magnet is a strong $\mathrm{Nd}$ $\mathrm{FeB}$ permanent magnet which is maintained on the rail, strongly enough to prevent the chain from moving in the leftward direction in the acceleration phase, but loosely enough to allow momentum propagation in the chain (a patch of putty can be observed in Figure 1). Note that the dipolar axis of the magnet is spontaneously aligned with the axis of the steel ball chain to minimize potential energy.

a) Electronic mail: nicolas.plihon@ens-lyon.fr

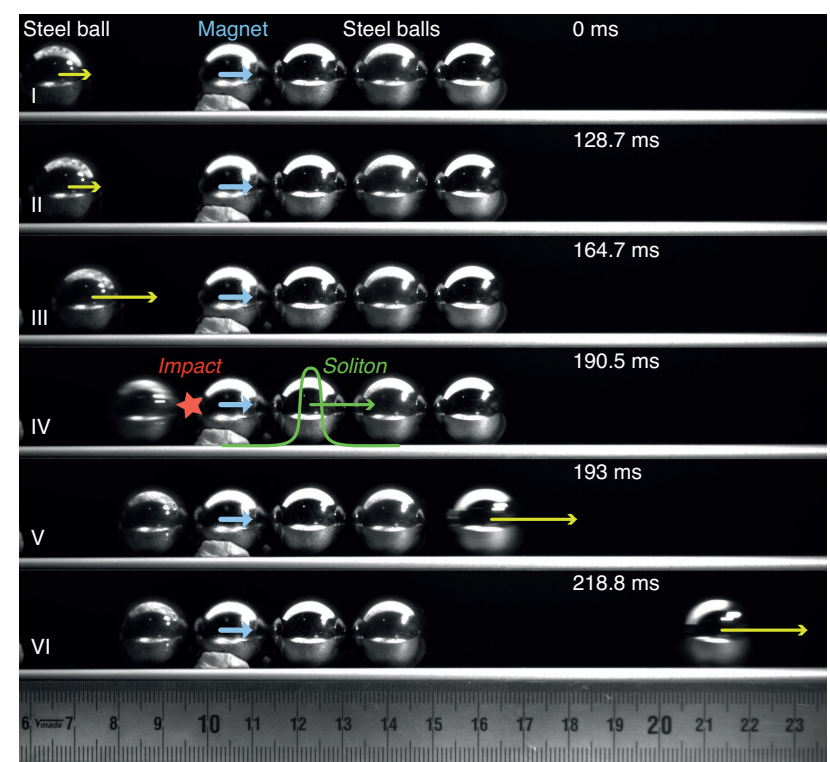

FIG. 1. Time sequence (from top to bottom) showing acceleration in the magnetic cannon. A line of three steel balls initially stuck to the right of a strong permanent magnet, loosely fixed on a rail, is impacted by a steel ball coming from the left hand side and accelerated in the magnetic field of the magnet. Momentum is transferred into the chain and the last ball on the right is ejected at high velocity. Images from a 2048x360 pixel film acquired at 400 frames per second.

The acceleration phase is governed by the magnetic field created by the magnet ${ }^{6,7}$ and the magnetization of the impacting steel ball. The determination of the magnetization of the incoming ferromagnetic steel ball differs from classical problems in which material (dia- or paramagnetic balls ${ }^{8}$ ) or geometry (large sample of ferromagnetic materials ${ }^{9-11}$ ) are different from ours. The gain of magnetic energy during the acceleration phase is called $U_{n}$ to indicate the dependence on the number of balls screening the magnetic field of the magnet. Part of this energy is converted into kinetic energy, which will add up to the initial kinetic energy of the incoming ball $K_{\text {init }}$, resulting in an impacting kinetic energy $K_{\text {impact }}$. The energy transfer in the chain is reminiscent of the Newton's cradle $^{12,13}$, governed by Hertzian contact forces involv- 


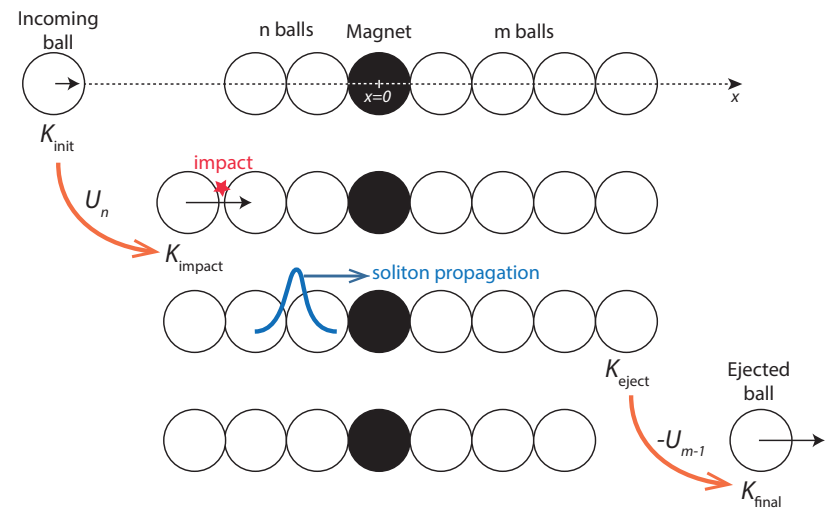

FIG. 2. Schematic of the sequences described in Figure 1 and associated kinetic $K$ and (potential) magnetic $U$ energies. Red arrows represent conversion between magnetic energy and kinetic energy.

ing dissipation ${ }^{14}$ in an inhomogeneous chain containing a magnet. Part of the impacting energy is transmitted through the chain, resulting in a kinetic energy $K_{\text {eject }}$. Finally, in the expulsion phase, the final kinetic energy of the ejected ball $K_{\text {final }}$ is given by the energy transmitted through the chain decreased by the energy required to escape the residual magnetic attraction on the righthand side of the chain, $U_{m-1}$. The goal of the present article is to relate $K_{\text {final }}$ to $K_{\text {init }}$ and the parameters of the system. Note that the energy conversion process at lead in this problem is not in contradiction with the fact that magnetic field do not work on charged particles (the Lorentz force being perpendicular to the charged particles velocity). The analysis of a situation similar to the one investigated here is discussed in details in Griffiths's textbook ${ }^{15}$; interested readers could also refer to the discussion about magnetic energy provided in Jackson's textbook ${ }^{16}$ (p. 224 and following) All effects related to the conversion of magnetic energy into kinetic energy are studied in section II. The transmission of kinetic energy through the chain is then detailed in section III. Finally, parameters influencing the global energy conversion of the system and the understanding of a succession of Gauss rifles are discussed in section IV.

\section{B. International Physicists Tournament}

The work presented here was done in preparation for the International Physicists Tournament (http:// iptnet.info/), a world-wide competition for undergraduate students. Each national team is composed of six students who work throughout the academic year on a list of seventeen open questions and present their findings during the tournament.

Unlike the typical physics exam, the problems must not only be presented, but also challenged and reviewed by the other participants allowing students to respectively assume the roles of researchers, referees and editors. In addition to the challenge that the tournament represents, it provides students with an exciting and eye-opening experience in which they learn how to design experiments with the aim of solving physics problems, and to constructively criticize scientific solutions.

The authors would highly recommend participation in the IPT as a rare learning opportunity for undergraduate students.

\section{What students can learn from this problem}

At introductory physics level, this experiment could be used to foster student's motivation while working on an open problem involving energy conservation. In a more classical laboratory work, students could reinvest their knowledge on magnetism to find out whether the incoming steel ball is to be considered as a permanent or an induced magnet through magnetic force and magnetic field measurements. At graduate level, the question of the dependence of the final velocity on some of the parameters of the system might lead to a few days experimental project work in which knowledge on mechanics, magnetism and non linear physics can be reused.

\section{FROM MAGNETIC ENERGY TO KINETIC ENERGY}

In this section we investigate the conversion of magnetic energy $U_{n}$ into kinetic energy in the acceleration phase, as well as the symmetric problem of the decrease of kinetic energy by $U_{m-1}$ in the ejection phase. Direct measurement of the spatial dependence of the magnetic force exerted by the magnet on the incoming (ejected) ball and of the magnetic field are in agreement with a permanent dipole/induced dipole modeling.

\section{A. Force exerted by the chain on a steel ball: a permanent/induced dipole interaction}

Let us first focus on the direct measurement of the magnetic force exerted on the steel ball. Its spatial evolution is measured using a weighing scale mounted as a Newton-meter, as sketched in Figure 3. A steel ball is attached to a heavy plastic block resting on the scale below the magnetic cannon chain. The steel ball attached to the scale is subject to its weight in the downward direction and to a magnetic force in the upward direction. The evolution of the force exerted by the magnet as a function of the distance $d$ between the center of the ball attached to the scale and the center of the magnet, derived from the apparent mass, is displayed in Figure 3. We observe that in the presence of one or two steel balls in front of the magnet, the force is screened but is nonetheless larger than it would have been with a chain of non-magnetic balls, since the ferromagnetic balls channel the magnetic field. A second observation, not illustrated here, is that 
the number of balls behind the magnet has no noticeable influence on the force exerted on the opposite side.
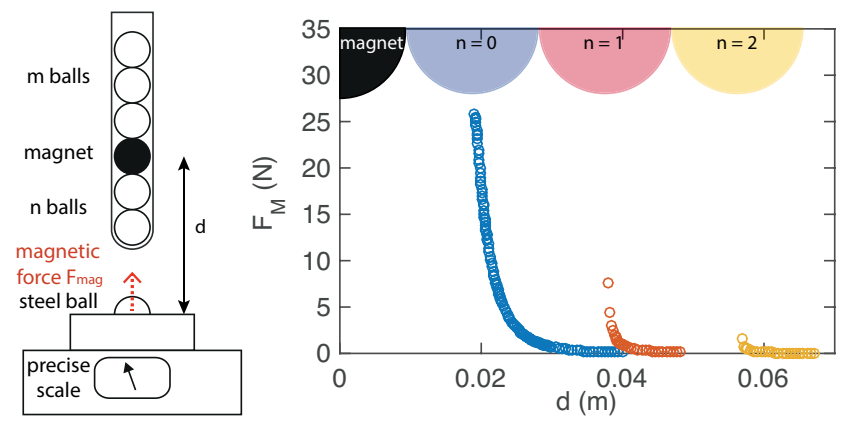

FIG. 3. Force measurement setup and spatial evolution of the magnetic force experienced by the steel ball attached to the scale as a function of the number $n$ of steel ball in front of the magnet.

We will now establish the relation between this magnetic force and the magnetic field created by the magnet, in a way similar to that developed by Jackson ${ }^{17}$, and show that the permanent/induced dipole assumption is accurate. The evolution of the intensity of the field along the magnet's axis as a function of the distance $d$ from its center is shown in Figure 4. The magnetic field intensity was measured using a Bell 7030 Gaussmeter but this could also be achieved using cheap and easy to implement

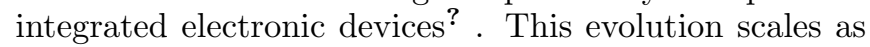
$d^{-3}$ as expected for a dipolar magnetic field. The magnetic field outside a uniformly magnetized sphere being exactly that of a point dipoleat the center of the sphere ${ }^{16}$, this scaling remains valid arbitrarily close to the magnet surface and the dipole strength of the magnet, $M_{0}$, can be determined according to

$$
B(d)=\frac{\mu_{0} M_{0}}{2 \pi d^{3}}
$$

where $\mu_{0}$ is the vacuum magnetic permeability. The best fits according to Equation 1 up to $d \sim 0.2 \mathrm{~m}$ are shown as full black lines in Figure 4 and lead to $M_{0}=3.64 \pm$ $0.1 \mathrm{Am}^{2}$.

The origin of the magnetic force experienced by the ferromagnetic steel ball lies in the interaction of the magnetic field created by the magnet and the magnetization of the steel ball. Assuming that the steel ball has an induced magnetic moment $\mathbf{m}_{\text {ball }}(\mathbf{d})$, the force reads

$$
\mathbf{F}_{\mathrm{M}}(d)=-\nabla\left(\mathbf{m}_{\text {ball }}(d) \cdot \mathbf{B}(d)\right)
$$

Equation 2 clearly shows a dependence on the magnetization properties of the steel ball. If $\mathbf{m}_{\text {ball }}(d)$ is constant and independent of $d$ (i.e. the steel is at saturation), $F_{\mathrm{M}}(d)$ is expected to scale as $d^{-4}$ as for an interaction between permanent dipoles ${ }^{6}$. On the other hand, if $\mathbf{m}_{\text {ball }}(d) \propto \mathbf{B}(d), F_{\mathrm{M}}(d)$ is expected to scale as $d^{-7}$ as for an interaction between a permanent dipole and an induced dipole.

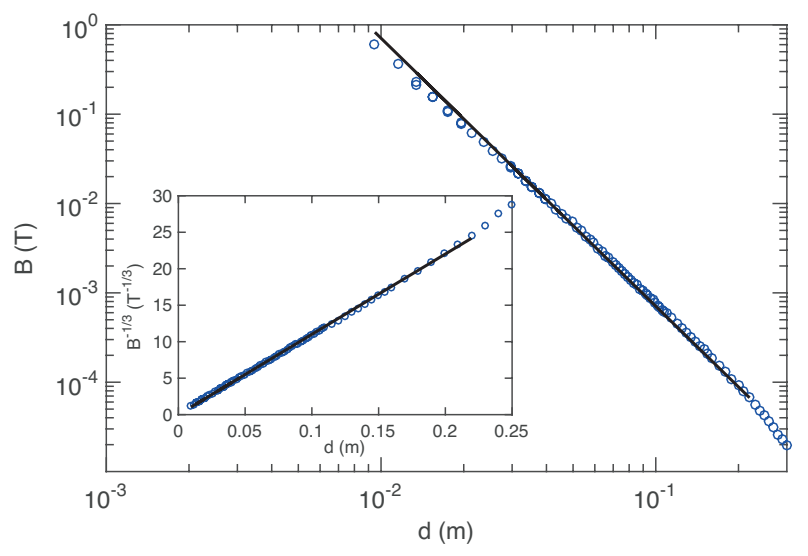

FIG. 4. Spatial evolution of the magnetic field created by the magnet - the inset shows $B^{-1 / 3}(d)$ - and associated dipolar best fits (black lines).

The experimental data presented in Figure 3 are displayed in logarithmic scales in Figure 5 and are consistent with a $d^{-7}$ scaling when $n=0$. This justifies the hypothesis of an induced magnetization proportional to the magnetic field, or equivalently a permanent/induced dipole interaction. A precise computation of the ball magnetization is a rather difficult task, since the field is highly inhomogeneous over the ball volume and the (unknown) magnetic permeability of the steel is expected to play a leading role. However, we will show in the following that a simple model correctly describes our experimental data. Let us first recall a classical result of magnetostatics (refer to p. 199 in textbook ${ }^{16}$ ) which gives the magnetic moment of a sphere of relative magnetic permeability $\mu_{r}$ immersed in a constant and homogeneous magnetic field $\mathbf{B}_{\mathbf{0}}$ as

$$
\mathbf{m}_{\mathrm{ball}}=\frac{4 \pi R^{3}}{3 \mu_{0}} \frac{3\left(\mu_{r}-1\right)}{\mu_{r}+2} \mathbf{B}_{\mathbf{0}}
$$

leading to a magnetic field intensity inside the ball $3 \mu_{r} B_{0} /\left(\mu_{r}+2\right)$. In other words the magnetic field is amplified inside the sphere, by a factor $3 \mu_{r} /\left(\mu_{r}+2\right)$. In the case of soft steel, one expects values of $\mu_{r}$ in the range $\left[50-10^{4}\right]$, which leads to a maximum three-fold increase. Let us make a crude approximation and now assume that Equation 3 remains valid in our configuration where the magnetic field created by the magnet is strongly inhomogeneous. Using the value of the magnetic field at the center of the steel ball, this leads to the following approximation of the magnetic force experienced by the steel ball:

$$
F_{\mathrm{M}}(d)=-\frac{4 \pi R^{3}}{\mu_{0}} \frac{\mu_{r}-1}{\mu_{r}+2} \frac{\partial B^{2}}{\partial d} \underset{\mu_{r} \gg 1}{\sim}-\frac{4 \pi R^{3}}{\mu_{0}} \nabla \frac{\partial B^{2}}{\partial d} .
$$

Using the dipolar model for the magnetic field (Equa- 
tion 1), the force can be conveniently expressed as:

$$
F_{\mathrm{M}}(d)=-\frac{6 \mu_{0} R^{3} M_{0}^{2}}{\pi d^{7}} \frac{\mu_{r}-1}{\mu_{r}+2} \underset{\mu_{r} \gg 1}{\sim}-\frac{6 \mu_{0} R^{3} M_{0}^{2}}{\pi d^{7}} .
$$

Figure 5 shows the spatial evolution of the force according to Equation 4 (black squares) and Equation 5 (solid black line) assuming $\mu_{r} \gg 1$. Our simple model is in very good agreement with the direct measurement of the force.

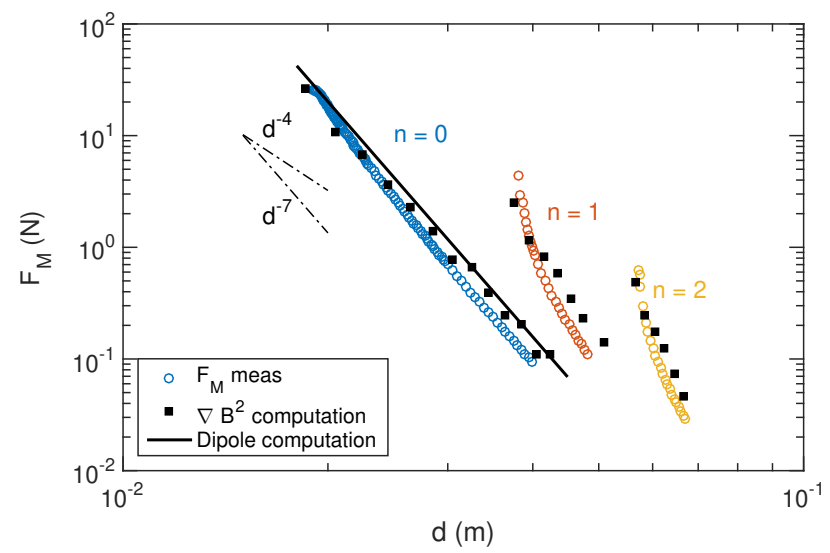

FIG. 5. Spatial evolution of the magnetic force for $n=0,1,2$ : direct force measurements (colored bullets), force computed from magnetic field measurements according to Equation 4 (black squares) and according to equation 5 for $n=0$ (solid black line) showing the validity of our permanent/induced dipole model.

When one or two balls are in front of the magnet (i.e. $n=1,2$ in Figure 5), establishing a theoretical expression of the magnetic field created by the magnet and channeled through the balls is beyond the scope of the present article. However, Figure 5 shows that the estimate of the magnetic force given by equation 4 is in close agreement with the direct measurement.

\section{B. Conversion of magnetic energy into kinetic energy}

The available magnetic energy in the presence of $n$ steel balls in front of the magnet is computed from the above spatial evolution of the magnetic force as $\int_{-\infty}^{2(n-1) R} F_{\mathrm{M}}(x) d x$; the upper limit of the integral being the minimum approaching distance of the center of the incoming ball from the center of the magnet. This can be done either by integration (i) of the measured force profile, (ii) of the force expressed as a function of the gradient of the square of the magnetic field profile according to equation 4 , which reads $U_{n}=4 \pi R^{3} B^{2}(2(n+1) R) / \mu_{0}$. Table I summarizes the estimations of the available magnetic energy according to these computations for several values of $n$. As expected from the previous subsection, a very good agreement is observed between these values, and the available magnetic energy can be conveniently computed from the magnetic field measurement.

\begin{tabular}{|l||c|c|c|}
\hline \hline$U_{n}$ & $n=0$ & $n=1$ & $n=2$ \\
\hline From force meas. & $72 \pm 3 \mathrm{~mJ}$ & $7.2 \pm 1 \mathrm{~mJ}$ & $1.4 \pm 0.2 \mathrm{~mJ}$ \\
\hline From field meas. & $75 \pm 25 \mathrm{~mJ}$ & $6.4 \pm 1 \mathrm{~mJ}$ & $1.6 \pm 0.4 \mathrm{~mJ}$ \\
\hline \hline
\end{tabular}

TABLE I. Available magnetic energy estimated from direct magnetic force measurement, or from a permanent/induced model involving the direct magnetic field measurement. The larger errors reported on last line lie in the low spatial resolution of the direct magnetic field measurements.

Note that a third computation of $U_{0}$ can also be performed by the integration of equation 5 when $n=0$ as $U_{0}=\mu_{0} M_{0}^{2} /\left(64 \pi R^{3}\right)=95 \pm 5 \mathrm{~mJ}$. This larger estimate can be understood from an overestimation of the magnetic field in the vicinity of the magnet using the dipolar approximation (as expected the dipolar approximation is not valid close to the magnet). Since most of the acceleration occurs very close to the magnet, this leads to an overestimate of $25 \%$ of the available magnetic energy. However, as shown below, the expression of the force given by equation 5 is useful to predict the time evolution of the speed of the impacting ball.

A partial conclusion can be drawn here for the optimization of the magnetic cannon. $U_{n}$ and $U_{m-1}$ represent respectively the gain and loss of magnetic (potential) energy. As $U_{k}$ strongly decreases with $k$, the maximum increase of magnetic energy is achieved for the lowest value value of $n$, i.e. $n=0$, while the minimum losses are obtained for large values of $m$. The optimization of the number $m$ of balls behind the magnet will be addressed in section IV.

Let us now consider the conversion of the available magnetic energy $U_{n}$ into kinetic energy. The incoming ball is subject to magnetic and friction forces. Friction forces may however be neglected: the magnetic force is of the order of $10 \mathrm{~N}$, while for $M=28 \mathrm{~g}$ balls and a maximum velocity of order of $3 \mathrm{~m} . \mathrm{s}^{-1}$, the friction force is estimated around $0.03 \mathrm{~N}$ and the viscous drag force around $10^{-4} \mathrm{~N}$. Because the magnetic force strongly increases as the distance between the incoming ball and the magnet decreases, most of the acceleration occurs in close vicinity of the magnet. As a consequence, the magnetic energy is mostly converted into translational energy and the rotation of the incoming ball can be neglected.

The work-energy theorem leads to an evolution of the ball velocity $\dot{x}$ as

$$
\dot{x}(x)=\sqrt{\frac{2}{M} \int_{-\infty}^{x} \vec{F}_{\mathrm{M}} \cdot \overrightarrow{\mathrm{d} x}+\dot{x}_{-\infty}^{2}}
$$

where $\dot{x}_{-\infty}$ is the initial velocity at large distance from the magnet (the initial velocity being null in the case of a single cannon, but may be non-zero when several successive rifles are investigated, as in subsection IV B). At 


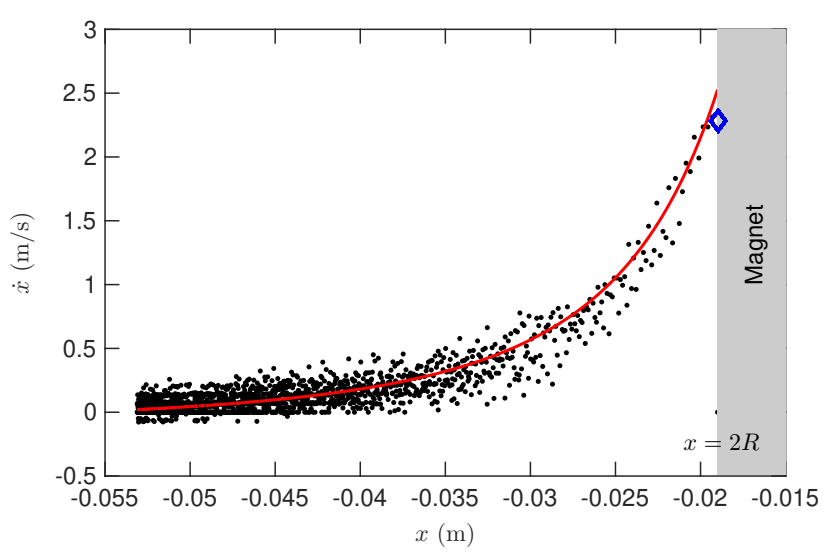

FIG. 6. Spatial evolution of the incoming ball velocity $(n=$ 0 ): experimental values from high-speed camera (dots), permanent/induced model (solid red curve) and final velocity estimated from integration of the magnetic force measurement, assuming a complete conversion of magnetic energy into kinetic energy (blue diamond).

the impact, this expression reads $K_{\text {impact }}=K_{\text {init }}+U_{n}$, i.e. a unity conversion factor. The assumptions leading to this simplified expression have been experimentally verified with $n=0$ and $\dot{x}_{-\infty}=0$. A high-speed camera (8000 frames per second) images the incoming ball during the acceleration phase. The velocity of the ball is computed as the derivative of the position of the center of the ball, extracted using Image J, a free software developed by NIH. Figure 6 displays the experimental velocity of the ball (dots) and the theoretical curve predicted by equation 6 using the dipole approximation of equation 5 (solid red line). The impact velocity computed from the integration of the direct force measurement (and assuming a complete conversion magnetic energy into kinetic energy) is also displayed as the blue diamond symbol. The good agreement of the measured final velocity with these estimates shows that friction may indeed be neglected and that the available magnetic energy is fully converted into translational kinetic energy. The red curve of Figure 6 shows that the permanent/induced dipole hypothesis enables to correctly predict the evolution of the impacting ball velocity; however, this model slightly overestimates the velocity, as expected from the overestimate of the available magnetic energy discussed above.

As a partial conclusion here, we showed that the whole available magnetic energy from the attraction of the magnet is converted into kinetic energy. Moreover, we provided a simplified expression of the force exerted by the magnet on the steel ball which leads to a theoretical expression of the magnetic energy $U_{n}$. Similar arguments can be applied in the ejection phase, where the kinetic energy of the ejected ball is given by $K_{\text {final }}=K_{\text {eject }}-U_{m-1}$.

\section{NESTERENKO SOLITON: FROM NEWTON'S CRADLE TO GAUSS CRADLE}

Following the impact of the incoming steel ball, the energy propagates in the ball chain similarly to what occurs in Newton's cradles ${ }^{12,13}$. However, in the magnetic cannon the chain is inhomogeneous (presence of a sintered $\mathrm{NdFeB}$ magnet). This section develops a classical model based on Hertzian contact and discusses briefly the Nesterenko soliton. Experimental yields accounting for the presence of the magnet are then presented.

\section{A. Nesterenko soliton: propagation of a non-linear wave}

Let us consider a chain of $N$ balls of radius $R$ allowed to translate along the $x$-axis and let the position of the balls be $x_{i}$ (see Figure 7 (a)). The force acting between two balls in contact is given by the Hertz law ${ }^{18}$ :

$$
F=\frac{E \sqrt{2 R}}{3\left(1-\nu^{2}\right)}\left(x_{i}-x_{i+1}-2 R\right)^{3 / 2}
$$

$E$ and $\nu$ being respectively the Young and Poisson moduli of the ball material.

In the case of the steel balls used in our experiments, an upper bound of the compression $\delta=x_{i}-x_{i+1}-2 R$ of the balls during the propagation of the wave can be estimated assuming equality of the compression energy $2 E \sqrt{2 R} \delta^{5 / 2} /\left(15\left(1-\nu^{2}\right)\right)$ with the kinetic energy of the impacting ball (of the order of $0.05 \mathrm{~J}$ ). The corresponding force is of the order of magnitude of $1000 \mathrm{~N}$. This largely exceeds the magnetic force experienced by the steel balls (even when in direct contact with the magnet, typically $25 \mathrm{~N}$ ). This demonstrates that the properties of the solitary compression wave are unaffected by the magnetic forces acting within the chain (although obviously the velocity of the impact ball strongly depends on the magnetic force).

Knowing the forces acting on each ball, the equations of motion can be numerically solved. Figure 7(b) shows the numerical solution for a chain of 5 steel balls $(E=210 \mathrm{GPa}, \nu=0.3)$ of radius $R=9.5 \mathrm{~mm}$. This graph shows several interesting features: (i) the final velocity of the impacting ball is negative, i.e. it experienced a rebound (note that unlike in the experiments, the first ball of the chain is unconstrained). (ii) The final velocity of the second-to-last ball is non-zero (although small), i.e. several balls can be ejected. Note that these features are visible in actual Newton's cradle. (iii) Since in this simple case the total energy is conserved, the velocity of the last ball is less than that of the impacting ball (roughly 98.7\%). The main conclusion is therefore that, even without any dissipation, the transmitted energy of the ejected ball $K_{\text {eject }}$ is lower than the kinetic energy of the impacting ball $K_{\text {impact }}$ and a yield $\eta=K_{\text {eject }} / K_{\text {impact }}$ should be introduced. For the specific case displayed in 
(a)

(b)
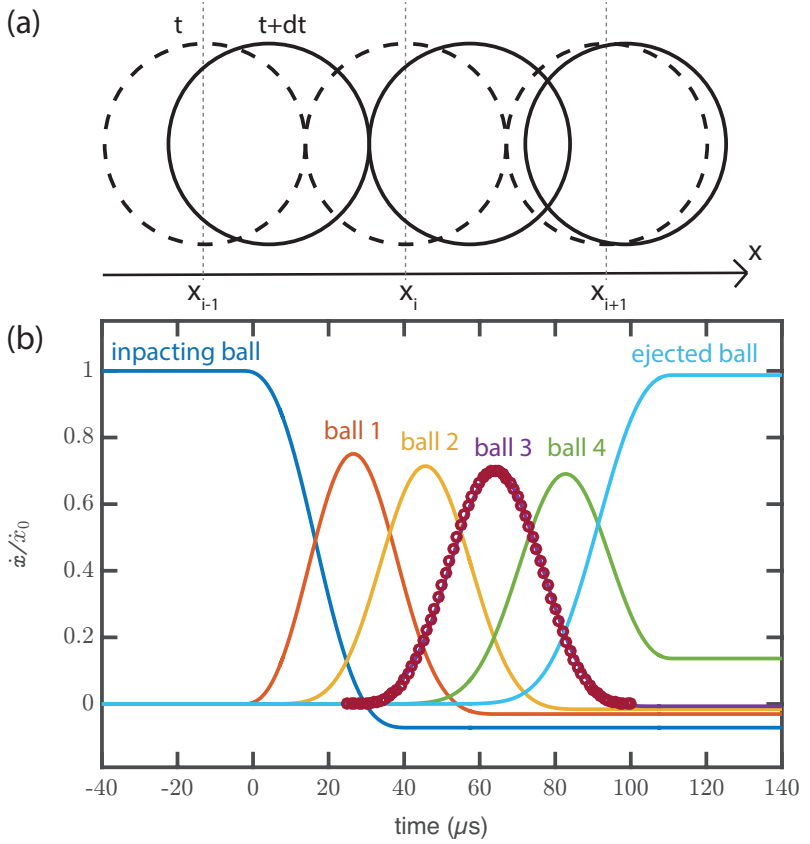

FIG. 7. Time evolution of the velocity of each ball normalized by the incoming velocity. The last ball is ejected at a slightly lower speed that the impacting ball and all other balls move. The open circles represent the analytic solution of the solitary wave.

Figure 7(b), the numerical simulation provides an estimate of $\eta=0.975$.

As a side-note, it is worth mentioning that an analytic solution to the continuous limit of the equation of motion was given by Nesterenko ${ }^{19,20}$ :

$$
\frac{\partial x}{\partial t}=A \sin ^{4}\left(\frac{x-c t}{L}\right) .
$$

This analytic solution is in excellent agreement with the numerical solution (see Figure 7). Remarkably the dispersion is counter-balanced by the non-linearities and this solitary wave travels without distortion. Among other interesting features, it should be mentioned that the spatial extension of the soliton is constant $(L \simeq 10 R)$ while its velocity $c$ increases with increasing amplitude $A$. The duration of the propagation for a given chain therefore decreases with increasing velocity of the impacting ball.

\section{B. Experimental measurement of the energy transmission yield $\eta$}

The above model does not account for any source of energy dissipation. An experimental determination of the yield $\eta=K_{\text {eject }} / K_{\text {impact }}$ is required to derive a global energy balance of the magnetic cannon. Figure 8(a) shows the experimental setup used: the energy of the impacting ball is controlled via the launching height of a pendulum hitting the chain (with no magnet) and the energy of the ejected ball is computed from its impact position on the ground after falling from a table. Similarly to the above mentioned model, none of the balls are fixed. Figure 8(b) shows the measurements of the energy transmitted to the ejected ball $K_{\text {eject }}$ as a function of $K_{\text {impact }}$. Several interesting features should be emphasized: (i) the energy transmitted to the ejected ball is proportional to the energy of the impacting ball, (ii) the yield $\eta$ decreases with the length of the chain, (iii) the experimental yield is lower than 0.975 due to the numerous sources of dissipation: for a chain of five balls, the experimental yield is only about 0.83 . The experimental determination of the yield is compatible with an evolution as $\eta=\eta_{0}-0.024(n+m+1)$, with $\eta_{0}=0.95$ in the case of a chain of steel balls. This result shows that dissipation sources (viscous and solid friction, deformations or imperfect contacts between the balls in the chain) cannot be neglected.

Moreover, in the magnetic cannon, the presence of the magnet does not only introduce an inhomogeneity, but also a magnetic field that magnetizes the steel balls, leading to a strong attraction between the balls - which prevents for instance the rebound of the impacting ball. The strength of the magnetic field does not modify the physical principles at lead in the Nesterenko soliton propagation: the maximum intensity of the magnetic force for a $600 \mathrm{mT}$ field, is three orders of magnitude below the mechanical compression forces. Moreover, the sintered $\mathrm{NdFeB}$ magnet introduces an inhomogeneity with distinct mechanical properties (Young and Poisson moduli and density) which may cause a higher dissipation due to the sintered structure of the magnet. The presence of an intruder (the magnet) in the chain also triggers a partial reflection of the wave. In order to estimate this effective dissipation, the experimental setup has been modified as represented in Figure 8(c). Figure 8(c) shows the evolution of the transmitted kinetic $K_{\text {trans }}$ as a function of the impacting kinetic energy $K_{\text {impact }}$ for a 7-balls chain with and without a magnet. Note that the increase/decrease of energy from magnetic acceleration/deceleration has been taken into account in the computation of the energies. Yet, the insertion of the magnet leads to an impressive yield drop from $81 \%$ to $44 \%$, leading to $\eta_{0} \sim 0.61$.

\section{OPTIMIZATION OF THE MAGNETIC CANNON}

\section{A. Optimization of a single magnetic cannon}

As previously stated, the optimal energy gain is obtained with no ball on the left of the magnet (or $n=0$ ), and strongly depends on the properties of the magnet. The optimal configuration also requires to lower the loss of magnetic energy $U_{m-1}$, which is obtained with a large number of balls on the right of the magnet (or $m \gg 1$ ), and to maximize the yield $\eta$ of the chain, which requires 

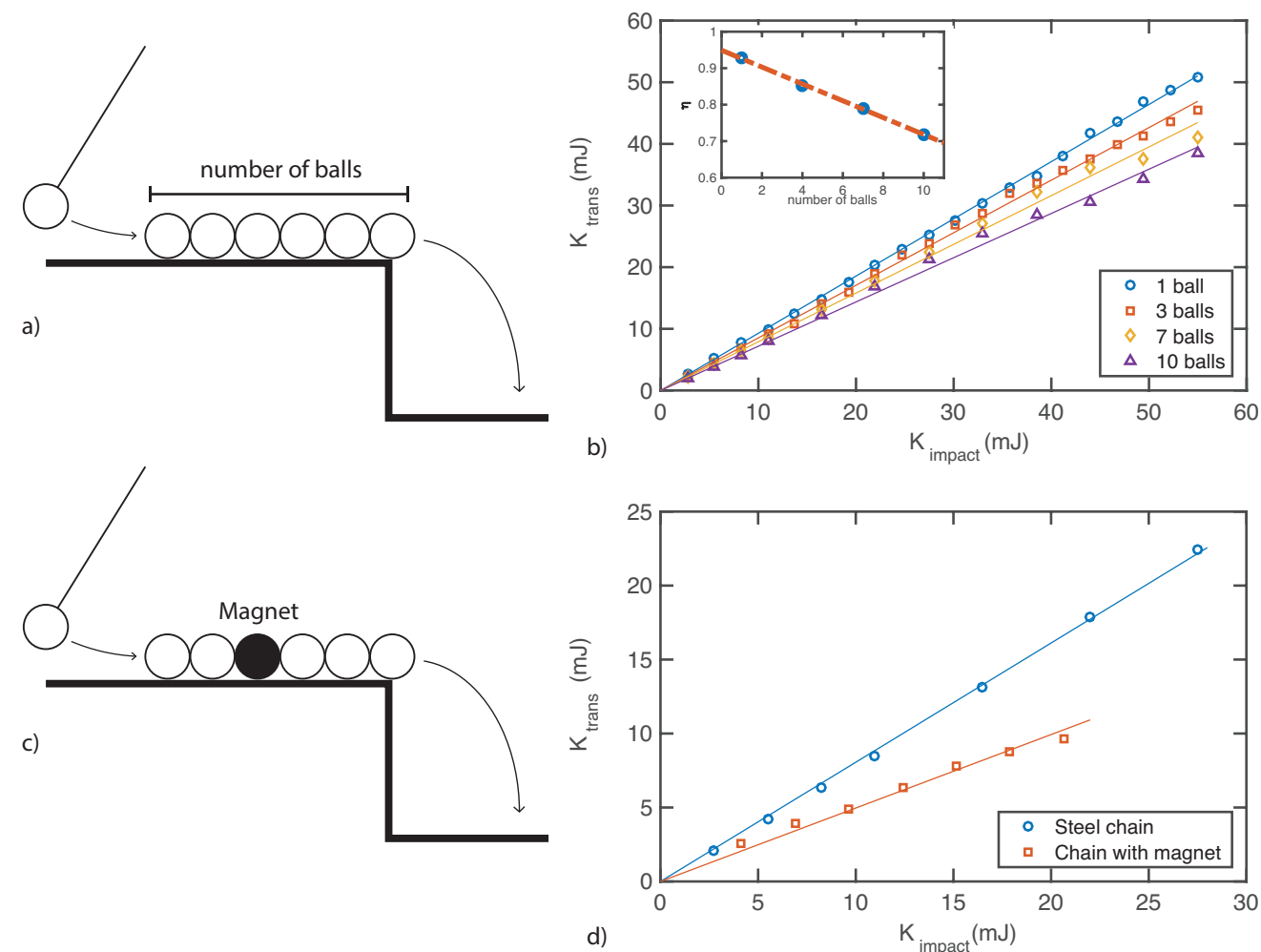

FIG. 8. (a) Diagram representing the setup for the energetic yield of the chain. The impacting energy is controlled thanks to a pendulum and the ejected energy is measured after a fall. (b) Energy of the ejected ball as a function of the energy of the impacting ball for different chain lengths without magnet. (c) Diagram representing the setup for the energetic yield of the chain with a magnet. (d) Energy of the ejected ball as a function of the energy of the impacting ball for a five balls length without magnet and with a magnet.

to minimize the total number $m+n+1$ of balls in the chain. The details provided in the previous sections enables us to express the kinetic energy of the ejected ball as a function of the initial kinetic energy and the properties of the system as

$$
K_{\text {final }}=\eta(n+m+1)\left[K_{\text {init }}+U_{n}\right]-U_{m-1}
$$

The maximal value of $K_{\text {final }}$ is obtained when the total losses [1- $\eta(n+m+1)] U_{n}+U_{m-1}$ are minimized. Figure 9 shows the evolution of the normalized losses as a function of $m$ for $n=0$ (in this configuration, no ball is ejected when $m=1$ ). A weak minimum is observed for $m=3$; for values of $m$ larger than 3, losses weakly increase with $m$. This Figure shows that the loss of magnetic energy $U_{m-1}$ can be neglected when $m>n+2$. The optimal configuration, i.e. maximizing the kinetic energy of the ejected ball, is thus obtained for no balls in front of the magnet and three balls behind the magnet (or $n=0, m=$ 3 ), which is the configuration displayed in Figure 1.

The kinetic energy of the ejected ball may be conveniently expressed for a spherical magnet of radius $R$ when neglecting $U_{m-1}$ in equation 7 . The dipolar moment of the magnet can be estimated as $M_{0} \sim 4 \pi R^{3} B_{r} /\left(3 \mu_{0}\right)$ with $B_{r}$ the residual flux density (of the order of $1.27 \mathrm{~T}$ for a grade $\mathrm{N} 40 \mathrm{NdFeB}$ magnet), which leads to

$$
K_{\text {final }} \sim\left(\eta_{0}-0.024(m+1)\right) \frac{\pi R^{3} B_{r}^{2}}{36 \mu_{0}}
$$

\section{B. Maximal acceleration achievable using $N$ successive rifles}

Once the optimization of one single magnetic rifle has been achieved, a natural question arises: to what extent is it possible to increase the ejected kinetic energy by using a succession of several rifles?

Let us now focus on a configuration with $N$ successive identical magnetic rifles: the ball ejected from rifle $i$ will be accelerated by rifle $i+1$ according to equation 7 . When neglecting losses between two successive rifles, the kinetic energy of the last ejected ball reads

$$
K_{\text {final }}(N)=\eta^{N} U_{\text {init }}^{0}+U_{n} \sum_{i=1}^{N} \eta^{i}-U_{m-1} \sum_{i=0}^{N-1} \eta^{i}
$$

The kinetic energy of the ejected ball increases with the number of modules, but since the energy gain is con- 


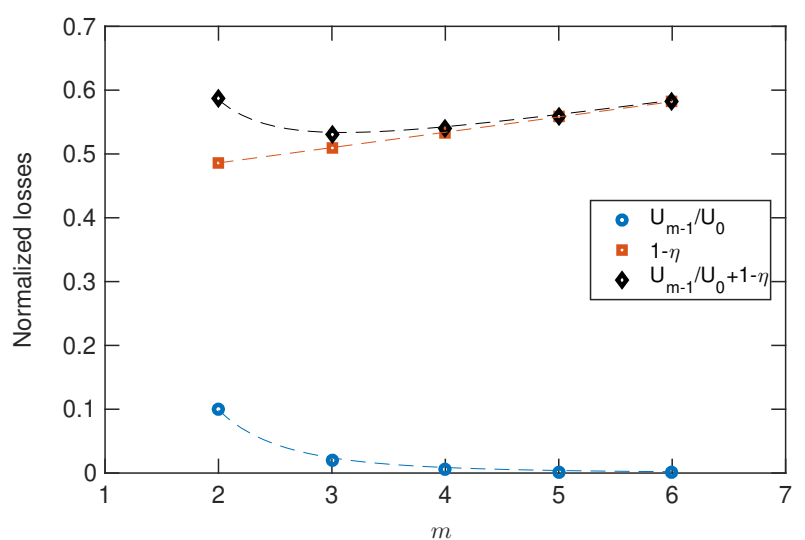

FIG. 9. Evolution of losses normalized to $U_{0}$ as a function of $m$ : loss of magnetic energy (blue circles), loss within the chain (red squares) and total losses (black diamonds).

stant and independent of the initial velocity while the dominant source of energy losses - through the propagation of the soliton within the chain - is proportional to the impacting energy, there is a maximum achievable kinetic energy

$$
K^{\max }=\frac{\eta U_{n}-U_{m-1}}{1-\eta}
$$

This saturation has been experimentally observed using a chain of 10 rifles composed of one magnet followed by $m=3$ balls and separated by $10 \mathrm{~cm}$ - note that for this specific setup, balls have a radius of $4 \mathrm{~mm}$. Velocities of the ejected ball were estimated from sound recording of the successive shocks between the ball ejected from rifle $i$ and the magnet of rifle $i+1$ and is displayed in Figure 10. This low-cost technique allows to provide the time-average velocity between two successive rifles. The experimental evolution is consistent with the prediction given above, neglecting $U_{m-1}$, using $U_{0}$ as determined by the direct force measurement and a yield $\eta=0.6$.

\section{v. CONCLUSION}

In conclusion we were able to successfully model the kinetic energy of the ball ejected from a magnetic cannon. In the acceleration phase, experimental data show a very good agreement with a simple model of uniform magnetization of a sphere plunged in the magnetic field created by the magnet. In the case where no steel balls are present between the incoming ball and the magnet, this magnetic field is accurately modeled as a dipolar magnetic field. We also provided a model based on Hertzian contact and solid collisions accounting for the propagation of momentum in the chain of balls and determined an

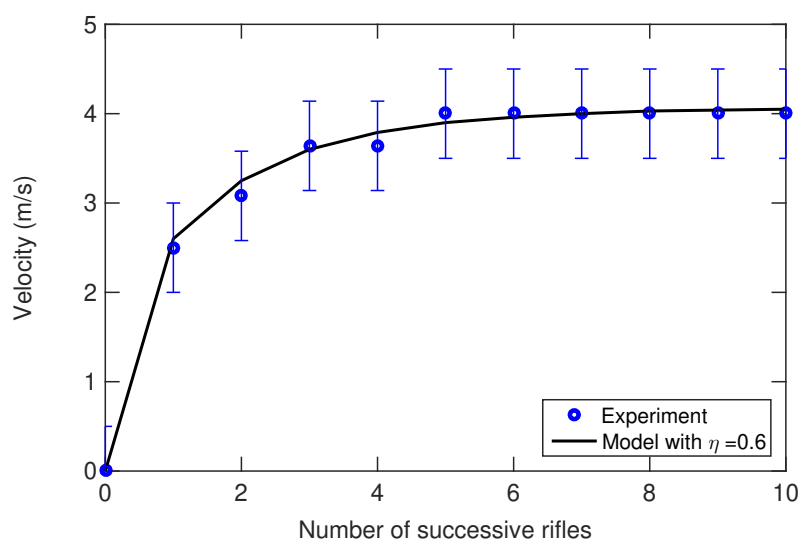

FIG. 10. Time-average velocity of balls ejected by successive identical rifles (estimated from sound recording) and model with $\eta=0.6$.

experimental effective yield accounting for its efficiency. These ingredients enable us to predict the final kinetic energy as a function of the parameters of the system (geometrical sizes, magnetic properties ) for a single magnetic cannon or an assembly of several modules.

Some limitations of our work could however deserve further analysis and modeling. In the presence of steel balls between the magnet and the incoming ball (i.e. $n \geq 1$ ), we derived the magnetic force as a function of the spatial evolution of the magnetic field. However a detailed modeling of the "channeling" of the dipolar field created by the magnet within ferromagnetic balls would make it possible to predict more precisely the energy gain in the acceleration phase. The study of the behavior of the magnetic cannon using paramagnetic or superparamagnetic materials instead of ferromagnetic materials could also be envisioned. The modeling of momentum propagation described in the present article is very similar to the Newton's cradle. Including the cohesion forces from the magnetic field as well as an effective behavior of the sintered NdFeB magnet could be an extension of this work.

\section{ACKNOWLEDGMENTS}

This work was supported by the École Normale Supérieure de Lyon and Univ Claude Bernard, Lyon, France. The organization committee of the International Physicist Tournament is gratefully acknowledged, as are contributions of the the French Academy of Sciences and all the other tournament's partners. We acknowledge also A. Bourges, C. Gouiller, A. Guittonneau, C. Malciu, G. Panel and J. Sautel with whom discussions and exchanges were prolific. 


\section{REFERENCES}

${ }^{1}$ J. A. Rabchuk. The Gauss Rifle and Magnetic Energy, Phys. Teach., 41, 158 (2003)

${ }^{2}$ D. Kagan. Energy and Momentum in the Gauss Accelerator, Phys. Teach., 42, 24 (2004)

${ }^{3}$ O. Chittasirinuwat, T. Kruatong and B. Paosawatyanyong. More fun and curiosity with magnetic guns in the classroom, Physics Education, 46, 318 (2011)

${ }^{4}$ C. Ucke and H.-J. Schlichting. Die Magnetkanone, Phys. Unserer Zeit, 40, 155 (2009)

${ }^{5}$ S. O. Starr, R. C. Youngquist, and R. B. Cox. A low voltage "railgun", Am. J. Phys., 81, 38 (2013)

${ }^{6}$ R. Castañer, J. M. Medina and M. J. Cuesta-Bolao. The magnetic dipole interaction as measured by spring dynamometers, Am. J. Phys., 74, 510 (2006)

${ }^{7}$ N. Derby and S. Olbert. Cylindrical magnets and ideal solenoids, Am. J. Phys., 78, 229 (2010)

${ }^{8}$ R. S. Davis. Using small, rare-earth magnets to study the susceptibility of feebly magnetic metals, Am. J. Phys., 60, 365 (1992)

${ }^{9}$ B. S. N. Prasad, S. V. Shastry and K. M. Hebbar. An Experiment to Determine the Relative Permeability of Ferrites, Am. J. Phys., 40, 907 (1972)

${ }^{10} \mathrm{~J}$. F. Borin and O. Baffa. Measuring magnetic properties of ferromagnetic materials, Am. J. Phys., 66, 449 (1998)

${ }^{11}$ W. M. Saslow. How a superconductor supports a magnet, how magnetically 'soft' iron attracts a magnet, and eddy currents for the uninitiated, Am. J. Phys., 59, 16 (1991)

${ }^{12}$ F. Herrmann and M. Seitz. How does the ball-chain work?, Am. J. Phys., 50, 977 (1982)

${ }^{13}$ S. Hutzler, G. Delaney, D. Weaire and F. MacLeod. Rocking Newton's cradle, Am. J. Phys., 72, 1508 (2004)

${ }^{14} \mathrm{D}$. Gugan. Inelastic collision and the Hertz theory of impact, $A m$. J. Phys., 68, 920 (2000)

${ }^{15}$ D. J. Griffiths. Introduction to Electrodynamics, fourth edition. Pearson ed (2013), pp 373-381

${ }^{16}$ J. D. Jackson. Classical Electrodynamics, third edition. John Wiley \& Sons ed (1998)

${ }^{17}$ D. P. Jackson. Dancing paperclips and the geometric influence on magnetization: A surprising result, Am. J. Phys., 74, 272 (2006)

${ }^{18} \mathrm{H}$. Hertz. On the contact of elastic solids, J. Reine Angew. Math., (1881)

${ }^{19} \mathrm{~S}$. Sen and M. Manciu. Discrete Hertzian chains and solitons, Physica A, 268, 644 (1999)

${ }^{20}$ V.F. Nesterenko. Propagation of nonlinear compression pulses in granular media, J. Appl. Mech. Tech. Phys., 5, 733 (1983) 